hep-th/9608065

\title{
A Locally Supersymmetric Action for the Scalar Particle
}

\author{
Fiorenzo Bastianelli周 and Luca Consolin \\ Dipartimento di Fisica, Universitá di Modena, via Campi 213/A, 41100 Modena, Italy
}

\begin{abstract}
We construct a locally supersymmetric action for the scalar particle, and study its relation with the usual reparametrization invariant action. The mechanisms at work are similar to those employed in the embedding of the bosonic string into the fermionic one, originally due to Berkovits and Vafa in their search for a universal string. The simpler algebraic structure present in the particle case provides us with a guide on how to prove in a simple way, without the need of fixing the superconformal gauge, that the supersymmetric formulation of the bosonic string is equivalent to the usual one, where reparametrization invariance is the only world-sheet gauge symmetry.
\end{abstract}

*email: bastianelli@imoax1.unimo.it

†email: consoli@imoax1.unimo.it 


\section{INTRODUCTION}

String theory is still lacking a suitable non-perturbative formulation. The best description available remains that of a perturbative expansion of strings propagating on a consistent background. String coordinates are quantized, and the different terms of the loop expansion are related to the different topologies of the world-sheet. In the absence of a more fundamental principle, different string theories have been classified according either to their world-sheet gauge symmetries or to their space-time properties. However, recent developments have made it clear that these two ways of classifying strings have not an intrinsic meaning; rather, they are seen to reflect properties of the chosen vacuum. In fact, on one hand string theories classified according to their space-time properties are related to each other by the so-called S, T and U dualities. This suggests that they may be interpreted as different perturbative expansions of a single unifying theory (the so-called M theory) about different vacua [1]. Similarly, in the other classification based on the world-sheet gauge symmetries (or, equivalently, on the constraint algebra of the conformal gauge formulation), it has been shown how the bosonic string described by a reparametrization invariant worldsheet action may be viewed as a special case of the fermionic string, which is described by a locally supersymmetric world-sheet action [2]. This hints on the possible existence of a universal string theory containing all the other ones as particular broken symmetric phases. In fact, in ref. [3] a hierarchy of supersymmetric strings was discovered, in which each string of the hierarchy contains also all those strings with a lower number of local supersymmetries on the world-sheet.

In this article we study the mechanism of embedding, originally discovered by Berkovits and Vafa, by applying it to a simpler case, namely the case of particles. A scalar particle is described by an action with reparametrization invariance, while the standard action for a

spin- $\frac{1}{2}$ particle has a local supersymmetry on the world-line [4]. We show that it is possible to construct a locally supersymmetric action describing the scalar particle. We solve the corresponding master equation of Batalin and Vilkovisky necessary for lagrangian quantization, and describe how this supersymmetric action is related to the usual reparametrization invariant one. We cast our discussion in the language of canonical transformations to exemplify that formalism. The simpler algebraic setting present in the particle case suggests how to proceed in the string case, and prove the equivalence of the locally supersymmetric formulation with the usual reparametrization invariant one, without the need of fixing the superconformal gauge. A simple field redefinition relates the local supersymmetric action given in [5] to the standard formulation of refs. [6]. This procedure bypasses subtleties of the superconformal gauge related to the treatment of the moduli and supermoduli of higher genus Riemann surfaces [7]. Eventually, we will present our conclusions.

\section{A RIGID SUPERSYMMETRIC MODEL}

We repeat in a simpler context the construction presented in [2]. In that reference Berkovits and Vafa found a suitable superconformal system that can be used as a background for the fermionic string in the superconformal gauge. The fermionic string propagating on such a background behaves exactly as the bosonic string. In a similar way, we will find a supersymmetric quantum mechanics that will reproduce the behaviour of a scalar particle 
once coupled to world-line supergravity. The supersymmetric action defining the model is given by

$$
S=\int d \tau\left(\frac{1}{2} \dot{x}^{\mu} \dot{x}_{\mu}+b_{1} \dot{c}_{1}\right)
$$

where $x^{\mu}$ are the coordinates of the particle, $\dot{x}^{\mu}=\frac{d}{d \tau} x^{\mu}$, and $\left(b_{1}, c_{1}\right)$ are a pair of anticommuting variables that will allow to realize supersymmetry. The rigid supersymmetry transformations are given by

$$
\begin{aligned}
\delta x^{\mu} & =\varepsilon c_{1} \dot{x}^{\mu} \\
\delta c_{1} & =\varepsilon+\varepsilon c_{1} \dot{c}_{1}, \\
\delta b_{1} & =-\varepsilon\left(\frac{1}{2} \dot{x}^{\mu} \dot{x}_{\mu}+\dot{b}_{1} c_{1}+2 b_{1} \dot{c}_{1}\right) .
\end{aligned}
$$

By computing the commutator algebra of two supersymmetry transformations one obtains a translation in the time parameter. This is the trademark of supersymmetry. The corresponding supersymmetry charge is

$$
Q=b_{1}-\frac{1}{2} c_{1} \dot{x}^{\mu} \dot{x}_{\mu}
$$

We note that the first term in the transformation of $c_{1}\left(\delta c_{1}=\varepsilon+\ldots\right)$ is the one that makes these rules different from the BRST rules arising after gauge-fixing the standard bosonic particle action

$$
S=\int d \tau \frac{1}{2} e^{-1} \dot{x}^{\mu} \dot{x}_{\mu}
$$

with the gauge condition $e=1$, and identifying the fields $c_{1}$ and $b_{1}$ with the corresponding ghost and antighost, respectively. This fact is also seen in the term proportional to $b_{1}$ appearing in the supersymmetry charge that makes it different from the BRST charge

$$
Q_{B R S T}=-\frac{1}{2} c_{1} \dot{x}^{\mu} \dot{x}_{\mu}
$$

Another observation is that the previous supersymmetry transformations can be simplified by adding suitable trivial symmetries proportional to the equations of motion, so to obtain

$$
\begin{aligned}
\delta x^{\mu} & =\varepsilon c_{1} \dot{x}^{\mu}, \\
\delta c_{1} & =\varepsilon \\
\delta b_{1} & =-\frac{1}{2} \varepsilon \dot{x}^{\mu} \dot{x}_{\mu} .
\end{aligned}
$$

However, with these simplified rules the supersymmetry algebra closes only on-shell on translations, while the previous rules close nicely off-shell.

As a final remark, we note that the supersymmetric action in eq. (2.1) can be generalized to a more general bosonic background described by a metric $g_{\mu \nu}(x)$, a vector potential $A_{\mu}(x)$ and a scalar potential $V(x)$, 


$$
S=\int d \tau\left(\frac{1}{2} g_{\mu \nu}(x) \dot{x}^{\mu} \dot{x}^{\nu}+A_{\mu}(x) \dot{x}^{\mu}+V(x)+b_{1} \dot{c}_{1}\right)
$$

The supersymmetry transformations are given now by

$$
\begin{aligned}
\delta x^{\mu} & =\varepsilon c_{1} \dot{x}^{\mu} \\
\delta c_{1} & =\varepsilon+\varepsilon c_{1} \dot{c}_{1}, \\
\delta b_{1} & =-\varepsilon\left(\frac{1}{2} g_{\mu \nu}(x) \dot{x}^{\mu} \dot{x}^{\nu}-V(x)+\dot{b}_{1} c_{1}+2 b_{1} \dot{c}_{1}\right) .
\end{aligned}
$$

Quantizing this system will give a supersymmetric quantum mechanics of a different type from the standard one [8]. However, it is a much less powerful supersymmetric quantum mechanics, since it is realized on a non-positive definite Hilbert space, being $\left(b_{1}, c_{1}\right)$ a system of ghost-like nature. In fact, many standard results will not apply (e.g. positive definiteness of the energy will not hold). This will not be a problem for our purposes since we plan to couple the model to gauge fields, those of world-line supergravity, allowing us to recover unitarity.

\section{A LOCALLY SUPERSYMMETRIC ACTION FOR THE SCALAR PARTICLE}

The system in (2.1) can be coupled to world-line supergravity. This will give a locally supersymmetric action, i.e. an action with the same local symmetries of the one describing the spin- $\frac{1}{2}$ particle. Using the Noether method to gauge the global symmetry in eq. (2.2), and dropping the space-time indices on the coordinates, we obtain

$$
S_{1}=\int d \tau\left(\frac{1}{2} e^{-1} \dot{x}^{2}+b_{1} \dot{c}_{1}+\chi b_{1}-\frac{1}{2} e^{-2} \chi c_{1} \dot{x}^{2}\right),
$$

where $e$ and $\chi$ are the einbein and the gravitino fields of the world-line, respectively. The local supersymmetry transformations are given by

$$
\begin{aligned}
\delta_{Q} x & =\varepsilon e^{-1} c_{1} \dot{x} \\
\delta_{Q} c_{1} & =\varepsilon+\varepsilon e^{-1} c_{1}\left(\dot{c}_{1}-\chi\right), \\
\delta_{Q} b_{1} & =-\frac{1}{2} \varepsilon e^{-2}\left(1-e^{-1} \chi c_{1}\right) \dot{x}^{2}-\varepsilon e^{-1} \dot{b}_{1} c_{1}-2 \varepsilon e^{-1} b_{1}\left(\dot{c}_{1}-\chi\right), \\
\delta_{Q} e & =2 \varepsilon \chi \\
\delta_{Q} \chi & =\dot{\varepsilon}
\end{aligned}
$$

while the reparametrizations are given by

$$
\begin{aligned}
\delta_{R} x & =\xi \dot{x}, \\
\delta_{R} c_{1} & =\xi \dot{c_{1}}, \\
\delta_{R} b_{1} & =\xi \dot{b_{1}}, \\
\delta_{R} e & =\partial_{\tau}(\xi e), \\
\delta_{R} \chi & =\partial_{\tau}(\xi \chi) .
\end{aligned}
$$


The transformation rules on the supergravity multiplet $(e, \chi)$ are the standard ones [4], and the algebra of local symmetries closes off-shell on all the fields

$$
\begin{aligned}
& {\left[\delta_{Q}\left(\varepsilon_{1}\right), \delta_{Q}\left(\varepsilon_{2}\right)\right]=\delta_{R}\left(\xi=2 \varepsilon_{2} \varepsilon_{1} e^{-1}\right)+\delta_{Q}\left(\varepsilon=-2 \varepsilon_{2} \varepsilon_{1} e^{-1} \chi\right),} \\
& {\left[\delta_{R}\left(\xi_{1}\right), \delta_{R}\left(\xi_{2}\right)\right]=\delta_{R}\left(\xi=\xi_{2} \dot{\xi}_{1}-\xi_{1} \dot{\xi}_{2}\right)} \\
& {\left[\delta_{R}\left(\xi_{1}\right), \delta_{Q}\left(\varepsilon_{2}\right)\right]=\delta_{Q}\left(\varepsilon=-\xi_{1} \dot{\varepsilon_{2}}\right) .}
\end{aligned}
$$

Because the complete algebra closes off-shell, it is straightforward to solve the BatalinVilkovisky master equation, useful for gauge-fixing in the lagrangian quantization [9]. The proper solution is given by

$$
\begin{aligned}
S_{1, B V} & =S_{1}+\int d \tau\left[x^{*}\left(\eta-\gamma e^{-1} c_{1}\right) \dot{x}+c_{1}^{*}\left(\dot{c}_{1} \eta+\gamma+\gamma e^{-1} c_{1}\left(\dot{c}_{1}-\chi\right)\right)\right. \\
& +b_{1}^{*}\left(\dot{b}_{1} \eta-\frac{1}{2} \gamma e^{-2}\left(1-e^{-1} \chi c_{1}\right) \dot{x}^{2}-\gamma e^{-1} \dot{b}_{1} c_{1}-2 \gamma e^{-1} b_{1}\left(\dot{c}_{1}-\chi\right)\right) \\
& \left.+e^{*}\left(\partial_{\tau}(\eta e)-2 \gamma \chi\right)+\dot{\chi}^{*}(\eta \chi-\gamma)+\eta^{*}\left(\dot{\eta} \eta+\gamma^{2} e^{-1}\right)+\gamma^{*}\left(\eta \dot{\gamma}-\gamma^{2} e^{-1} \chi\right)\right],
\end{aligned}
$$

where $\eta$ and $\gamma$ are the ghosts for $\xi$ and $\varepsilon$, respectively, and the starred fields are the so-called antifields (i.e. the sources for the BRST variations).

To see why this action describes a scalar particle, we note that variables can be redefined and that the basis of gauge symmetries is not unique, since it can be modified considerably by adding trivial gauge symmetries proportional to the equations of motions. In fact, after a little inspection, we see that by defining the new variables

$$
\begin{aligned}
& \tilde{e}=e+\chi c_{1}, \\
& \tilde{\chi}=\chi-\dot{c}_{1},
\end{aligned}
$$

the action (3.1) can be rewritten as follows

$$
S_{2}=\int d \tau\left(\frac{1}{2} \tilde{e}^{-1} \dot{x}^{2}+\tilde{\chi} b_{1}\right)
$$

Moreover, the two gauge symmetries can also be represented by

$$
\begin{aligned}
\delta x & =\tilde{\xi} \dot{x}, \\
\delta c_{1} & =\tilde{\varepsilon}, \\
\delta b_{1} & =0, \\
\delta \tilde{e} & =\partial_{\tau}(\tilde{\xi} \tilde{e}), \\
\delta \tilde{\chi} & =0,
\end{aligned}
$$

where $\tilde{\xi}$ and $\tilde{\varepsilon}$ are the two independent gauge parameters. We see immediately that the variable $c_{1}$ can be dropped since it is a pure gauge degree of freedom, while the variables $b_{1}$ and $\tilde{\chi}$ are non-dynamical fields which can be eliminated by their equations of motion. Thus, we are left with the standard reparametrization invariant action describing a massless scalar particle, and quantization can proceed in the well-known way. This proves our claim that a scalar particle can be described by a locally supersymmetric action. Note that the gauge symmetries in eqs. (3.2) and (3.3), when expressed in terms of the new variables, can be written as follows 


$$
\begin{aligned}
\delta x & =\tilde{\xi} \dot{x} \\
\delta c_{1} & =\tilde{\varepsilon}+\tilde{\xi} \dot{c}_{1}, \\
\delta b_{1} & =\tilde{\xi} \partial_{\tau} \frac{\delta_{L} S_{2}}{\delta \tilde{\chi}}+\tilde{\varepsilon} \frac{\delta S_{2}}{\delta \tilde{e}}-2 \tilde{\varepsilon} \tilde{e}^{-1} b_{1} \frac{\delta_{L} S_{2}}{\delta b_{1}}, \\
\delta \tilde{e} & =\partial_{\tau}(\tilde{\xi} \tilde{e})-\tilde{\varepsilon} \frac{\delta_{L} S_{2}}{\delta b_{1}}, \\
\delta \tilde{\chi} & =-\partial_{\tau}\left(\tilde{\xi} \frac{\delta_{L} S_{2}}{\delta b_{1}}\right),
\end{aligned}
$$

where we have defined for convenience

$$
\begin{aligned}
& \tilde{\xi}=\xi+\varepsilon e^{-1} c_{1}, \\
& \tilde{\varepsilon}=\varepsilon-\varepsilon e^{-1} c_{1} \chi,
\end{aligned}
$$

and where $\frac{\delta_{L} S}{\delta \phi}$ denotes the left functional derivative of the action. Dropping the terms proportional to the equations of motion from the right hand side, and shifting $\tilde{\varepsilon} \rightarrow \tilde{\varepsilon}-\tilde{\xi} \dot{c}_{1}$ one obtains the simplified basis of gauge symmetries. Thus, we see how the simple gauge symmetries in eq. (3.8), containing the reparametrizations and a shift symmetry on a fermionic variable, can mimic the full transformations of local supersymmetry.

The proper solution of the master equation for the action in (3.7) with gauge symmetries in (3.8) can be obtained quite easily. Dropping the tilde on the variables we have

$$
S_{2, B V}=\int d \tau\left(\frac{1}{2} e^{-1} \dot{x}^{2}+\chi b_{1}+x^{*} \eta \dot{x}+e^{*} \partial_{\tau}(\eta e)+c_{1}^{*} \gamma+\eta^{*} \dot{\eta} \eta\right) .
$$

The actions $S_{1, B V}$ and $S_{2, B V}$ describe the same model and are both proper solutions of the master equation. A theorem guarantees that these actions must be related by canonical transformations. In the Batalin-Vilkovisky formalism, canonical transformations are typically used to gauge-fix and to redefine variables (in fact, the process of gauge-fixing can be thought of as a particular field redefinition). They preserve the properties of the antibracket and map proper solutions into proper solutions. Canonical transformations are specified by a fermionic generating function $\Psi$ and are defined by $\phi \rightarrow \phi^{\prime}=e^{\mathcal{L}_{\Psi}} \phi$, where $\phi$ is a field or an antifield, and $\mathcal{L}_{\Psi} \phi \equiv(\Psi, \phi)$ with $($,$) denoting the antibracket. The canonical$ transformation between $S_{1, B V}$ and $S_{2, B V}$ can be presented in a factorized form

$$
S_{2, B V}=e^{\mathcal{L}_{\Psi_{7}}} e^{\mathcal{L}_{\Psi_{6}}} e^{\mathcal{L}_{\Psi_{5}}} e^{\mathcal{L}_{\Psi_{4}}} e^{\mathcal{L}_{\Psi_{3}}} e^{\mathcal{L}_{\Psi_{2}}} e^{\mathcal{L}_{\Psi_{1}}} S_{1, B V}
$$

where the various gauge fermions are given by

$$
\begin{aligned}
& \Psi_{1}=\int d \tau e^{*} \chi c_{1}, \\
& \Psi_{2}=-\int d \tau \chi^{*} \dot{c}_{1}, \\
& \Psi_{3}=-\int d \tau \eta^{*} \gamma e^{-1} c_{1}, \\
& \Psi_{4}=-\int d \tau \gamma^{*} \gamma e^{-1} c_{1}\left(\dot{c}_{1}+\chi\right), \\
& \Psi_{5}=\int d \tau\left[e^{*} \chi c_{1}+b_{1}^{*} c_{1}\left(\frac{1}{2} e^{-2} \dot{x}^{2}-2 e^{-1} b_{1} \chi\right)\right],
\end{aligned}
$$




$$
\begin{aligned}
& \Psi_{6}=-\int d \tau \dot{\chi}^{*} b_{1}^{*} \eta \\
& \Psi_{7}=-\int d \tau \gamma^{*} \eta \dot{c_{1}} .
\end{aligned}
$$

We note that the transformations specified by $\Psi_{1}$ and $\Psi_{2}$ accomplish the field redefinition given in eq. (3.6), $\Psi_{3}$ and $\Psi_{4}$ redefine the ghosts (i.e. the gauge parameters) as in eq. (3.10), $\Psi_{5}$ and $\Psi_{6}$ modify the gauge symmetry basis by terms proportional to the equations of motion as in eq. (3.9), and $\Psi_{7}$ performs a final redefinition of the ghost $\gamma$.

In ref. [5], the same method of canonical transformations was used to prove the equivalence between the fermionic string propagating on the Berkovits-Vafa background and the bosonic string. However, that proof was given in the conformal gauge, as was the original proof in [2] and the conformal field theoretical proof in [10]. Here, in the simpler case of particles, we have been able to treat the geometrical fields on the world-line, the einbein and the gravitino, in their full generality, without the need of specifying a gauge condition. The lesson learned in this simple model will help us in re-examining the string case from a better perspective.

Before closing this section, we note that it is straightforward to generalize the supersymmetric action for the particle by including a mass term, and, in general, the full background described in eq. (2.7) (a constant term in the potential $V$ can reproduce the effect of a mass term for the particle). For simplicity we have discussed here only the basic case of a massless particle propagating on a flat space-time.

\section{EQUIVALENCE OF THE FERMIONIC STRING ON THE BERKOVITS-VAFA BACKGROUND AND THE BOSONIC STRING}

We have seen in the previous section how a locally supersymmetric formulation of the scalar particle can be related to the standard reparametrization invariant one. The relation is given by a field redefinition consisting in a shift of the gravitino field, and in a certain transformation on the einbein. The latter can also be interpreted as a particular supersymmetry transformation. Guided by this particular field redefinition, we will relate the locally supersymmetric formulation of the bosonic string to the usual reparametrization invariant one. The locally supersymmetric action, which in the superconformal gauge reproduces the model of Berkovits and Vafa, was constructed in ref. [5], and reads:

$$
\begin{aligned}
S=\frac{1}{\pi} \int d^{2} x e( & \frac{1}{2} \nabla_{+} X \nabla_{=} X+b_{1} \nabla_{=} c_{1}+\bar{b}_{1} \nabla_{+} \bar{c}_{1}+\chi_{-=} G_{++}+\chi_{++} G_{-=} \\
& \left.+\chi_{++} \chi_{-=} c_{1} \bar{c}_{1} \nabla_{+} X \nabla_{=} X\right)
\end{aligned}
$$

where

$$
\begin{aligned}
& G_{++}=b_{1}\left(1+c_{1} \nabla_{+} c_{1}\right)-\frac{1}{2} c_{1}\left(\nabla_{+} X\right)^{2} \\
& G_{-=}=\bar{b}_{1}\left(1+\bar{c}_{1} \nabla_{=} \bar{c}_{1}\right)-\frac{1}{2} \bar{c}_{1}\left(\nabla_{=} X\right)^{2}
\end{aligned}
$$

and where $\left(b_{1}, c_{1}, \bar{b}_{1}, \bar{c}_{1}\right)$ are Lorentz tensors with spin $\left(\frac{3}{2},-\frac{1}{2},-\frac{3}{2}, \frac{1}{2}\right), \nabla_{a}=e_{a}{ }^{\mu} \partial_{\mu}+\omega_{a} J$ is the Lorentz covariant derivative with the flat index $a$ taking values $(+,=), J$ is the Lorentz 
generator which measures the Lorentz spin, and $\omega_{a}$ is the spin connection (see ref. [5] for more extended definitions). The following redefinition of variables

$$
\begin{aligned}
\tilde{e}_{+}{ }^{\mu} & =e_{+}{ }^{\mu}-\chi_{++} \bar{c}_{1} e_{=}{ }^{\mu}, \\
\tilde{e}_{=}{ }^{\mu} & =e_{=}{ }^{\mu}-\chi_{-=} c_{1} e_{+}{ }^{\mu}, \\
\tilde{\chi}_{++} & =e\left(\chi_{++}+\chi_{++} \bar{c}_{1} \nabla_{=} \bar{c}_{1}-\nabla_{+} \bar{c}_{1}\right), \\
\tilde{\chi}_{-=} & =e\left(\chi_{-=}+\chi_{-=} c_{1} \nabla_{+} c_{1}-\nabla_{=} c_{1}\right),
\end{aligned}
$$

brings the action (4.1) in the form

$$
S=\frac{1}{\pi} \int d^{2} x\left(\frac{\tilde{e}}{2} \tilde{\nabla}_{+} X \tilde{\nabla}_{=} X+\tilde{\chi}_{-=} b_{1}+\tilde{\chi}_{++} \bar{b}_{1}\right) .
$$

Once more, the fields $\left(c_{1}, \bar{c}_{1}\right)$ can be dropped since the action does not depend on them, and so they are pure gauge degrees of freedom, while $\left(b_{1}, \tilde{\chi}_{-=}\right)$and $\left(\bar{b}_{1}, \tilde{\chi}_{+++}\right)$are non-dynamical auxiliary fields which can be eliminated by their equations of motion. This leaves us with the standard action for the bosonic string, written here using the world-sheet vielbein rather than the world-sheet metric. At this point, one could also discuss how the supersymmetry transformations for the action (4.1), given in [5], are mimicked by the natural gauge symmetries of eq. (4.4), reparametrizations and local shifts in $\left(c_{1}, \bar{c}_{1}\right)$. However, we leave this as an exercise, since it is quite similar to the particle case discussed above.

The advantage of our proof of the equivalence between the fermionic string on the Berkovits-Vafa background and the bosonic string is that we didn't have to fix the conformal gauge. The latter is typically too strong a condition on the geometrical fields of the world-sheet, and modular integrations corresponding to the antighosts zero modes must be performed. We recognize that, after fixing the conformal gauge for the supersymmetric model discussed above, one would get integrations over the moduli corresponding to the gravitino, but these would be just gauge artifacts. Our proof dispenses us from analyzing how the particular mechanism for these modular integrations would work out.

\section{CONCLUSIONS}

We have constructed and analyzed a locally supersymmetric formulation for the scalar particle. This model employes in a simple context the mechanism discovered by Berkovits and Vafa for embedding the bosonic string into the fermionic one. Guided by the particle model we have been able to give a simpler proof of the string embedding. String theory is supposed to determine its own background, so such an embedding is taken as an indication of the existence of a "universal string theory". However, this is a non-perturbative statement, and very little information can be extracted by the existence of the above embedding. In fact, as we have seen, properties arising from such embeddings are essentially gauge artifacts. In contrast, the other hint about the existence of a unifying string theory, which comes from $\mathrm{S}, \mathrm{T}$ and $\mathrm{U}$ dualities, has been much more fruitful, since it has a lot of predictive power [1]. One should remember that any gauge algebra can always be brought into an abelian form [11]. Sometimes, this clashes with manifest locality and/or manifest covariance with respect to certain symmetries (like Lorentz covariance in standard Yang-Mills theory), and thus the non-abelian basis may be a preferred one. As we have seen, in the first quantized description of particles and strings, there does not seem to exist a preferred basis for the gauge symmetries that can be used for an intrinsic definition of the various models. 


\section{REFERENCES}

[1] see e.g. J.H. Schwarz, "The Second Superstring Revolution”, hep-th/9607067; "Lectures on Superstring and M Theory Dualities", hep-th/9607201.

[2] N. Berkovits and C. Vafa, hep-th/9310170, Mod. Phys. Lett.A9, 653 (1994).

[3] F. Bastianelli, N. Otha and J.L. Petersen, hep-th/9403150, Phys. Rev. Lett. 73, 1199 (1994).

[4] L. Brink, S. Deser, B. Zumino, P. Di Vecchia and P. Howe, Phys. Lett. B64, 435 (1976).

[5] F. Bastianelli, hep-th/9311157, Phys. Lett. B322, 340 (1994).

[6] L. Brink, P. Di Vecchia and P. Howe, Phys. Lett. B65, 471 (1976);

S. Deser and B. Zumino, Phys. Lett. B65, 369 (1976).

[7] see e.g. comments at page 90 of J. Polchinski's 1994 Les Houches Summer School Lectures "What is String Theory?", hep-th/9411028.

[8] E. Witten, Nucl. Phys. B118, 513 (1981).

[9] I. A. Batalin and G. A. Vilkovisky, Phys. Lett. B102 27 (1981); for a review see e.g. J. Gomis, J. París and S. Samuel, hep-th/9412228, Phys. Rep. 259, 1 (1995).

[10] I. Ishikawa and M. Kato, hep-th/9311139, Mod. Phys. Lett. A9 (1994) 725.

[11] I. A. Batalin and G. A. Vilkovisky, Nucl. Phys. B234 106 (1984). 